Article

\title{
Variation in Gas and Volatile Compound Emissions from Human Urine as It Ages, Measured by an Electronic Nose
}

\author{
Siavash Esfahani ${ }^{1,+}{ }^{\dagger}$, Nidhi M. Sagar ${ }^{2,+}$, Ioannis Kyrou ${ }^{3}$, Ella Mozdiak ${ }^{2}$, Nicola O'Connell ${ }^{4}$, \\ Chuka Nwokolo $^{4}$, Karna D. Bardhan ${ }^{5,6}$, Ramesh P. Arasaradnam ${ }^{4,6}$ and James A. Covington ${ }^{1, *}$ \\ 1 School of Engineering, University of Warwick, Coventry, CV4 7AL, UK; s.esfahani@warwick.ac.uk \\ 2 School of Medicine, University of Warwick, Coventry, CV4 7AL, UK; dr.nidhisagar@gmail.com (N.M.S.); \\ ella.mozdiak@nhs.net (E.M.) \\ 3 Department of Metabolic Medicine, University Hospital Coventry and Warwickshire, Coventry, \\ CV2 2DX UK; I.Kyrou@warwick.ac.uk \\ 4 Department of Gastroenterology, University Hospital Coventry and Warwickshire, Coventry, CV2 2DX, UK; \\ Nicola.Oconnell@uhcw.nhs.uk (N.O.C.); chukka.nwokolo@uhcw.nhs.uk (C.N.); \\ R.Arasaradnam@warwick.ac.uk (R.P.A.) \\ 5 Rotherham General Hospital, Rotherham, S60 2UD, UK; bardhan.sec@rothegn.nhs.uk \\ 6 Clinical Sciences Research Institute, University of Warwick, Coventry, CV2 2DX, UK \\ * Correspondence: j.a.covington@warwick.ac.uk; Tel.: +44-247-657-4494; Fax: +44-247-641-8922 \\ + These authors contributed equally to this work.
}

Academic Editor: Nastaran Hashemi

Received: 29 November 2015; Accepted: 20 January 2016; Published: 25 January 2016

\begin{abstract}
The medical profession is becoming ever more interested in the use of gas-phase biomarkers for disease identification and monitoring. This is due in part to its rapid analysis time and low test cost, which makes it attractive for many different clinical arenas. One technology that is showing promise for analyzing these gas-phase biomarkers is the electronic nose-an instrument designed to replicate the biological olfactory system. Of the possible biological media available to "sniff", urine is becoming ever more important as it is easy to collect and to store for batch testing. However, this raises the question of sample storage shelf-life, even at $-80^{\circ} \mathrm{C}$. Here we investigated the effect of storage time (years) on stability and reproducibility of total gas/vapour emissions from urine samples. Urine samples from 87 patients with Type 2 Diabetes Mellitus were collected over a four-year period and stored at $-80^{\circ} \mathrm{C}$. These samples were then analyzed using FAIMS (field-asymmetric ion mobility spectrometry-a type of electronic nose). It was discovered that gas emissions (concentration and diversity) reduced over time. However, there was less variation in the initial nine months of storage with greater uniformity and stability of concentrations together with tighter clustering of the total number of chemicals released. This suggests that nine months could be considered a general guide to a sample shelf-life.
\end{abstract}

Keywords: urinary stability; storage; electronic nose; ion mobility spectrometry; headspace analysis

\section{Introduction}

There is an increasing medical interest in the detection and monitoring of gases and volatiles for the identification and monitoring of disease. One technology that has shown promise in detecting these gases and volatiles is the electronic nose. Since its invention in the 1980s [1] it has seen considerable use with the medical field and has been applied to diseases as diverse as brain cancers to tuberculosis and even wound monitoring [2-4]. Such instruments do not attempt to detect individual chemicals in a sample but, like the biological nose, attempt to analyze a sample as a whole by using an array 
of non-selective chemical sensors. Its clinical diagnostic utility has a number of key advantages over many other current techniques; it is a low-cost test, the liquid/solid phase sample does not need to enter the instrument, there are no reagents involved and the results can be provided in almost real-time, making it attractive as a future point-of-care tool [5-9].

Our group has shown the utility of the electronic nose to detect and monitor a range of gastroenterological and metabolic disorders $[10,11]$. We have focused primarily on investigating urine as the biological waste of choice, as it provides an integrated physiological signal that is stable, can be provided on demand and, in our experience, is the most "user friendly" of biological samples for both the patient and the investigation team when compared with blood, breath and faeces. However, in ours and many other urinary based studies, it can be difficult to analyse chemical signals from samples at the point of collection. The reasons for this are many, but usually due to a combination of the disease having low-prevalence combined with the logistical advantages (in terms of time, money and access to equipment) of batch processing. In this case freezing of the sample (normally at $-80^{\circ} \mathrm{C}$ ) is undertaken. Thus an important question that has concerned us, and probably others, is "what is the best before date for a urine sample?". Due to the way in which electronic noses analyse samples, it is very challenging to monitor the degradation of any specific biomarker. However, it should be possible to be guided on what a sensible storage period might be by understanding the general loss of chemical information (or by the way that the urine sample changes over time). Therefore, in this study we have attempted to understand the stability and degradation of a urine samples, using the electronic nose as the measurement technology.

Previous work for urine stability has focused almost solely in the liquid phase, applying techniques such as NMR (nuclear magnetic resonance), GC-TOF-MS (gas chromatography-time of flight-mass spectrometry) and LC-MS (liquid chromatography-mass spectrometry) to investigate this problem [12-14]. However, as this does not employ headspace analysis (using an electronic nose or other approach), its relevance is low. Other groups have also undertaken headspace analysis of urine samples to investigate the natural variation by GCMS (gas chromatography/mass spectrometry) and SIFT-MS (selective ion flow tube-mass spectrometry), but did not consider stability of their samples $[15,16]$. There have been a small number of studies using GCMS investigating sample age over $24 \mathrm{~h}$ in humans and in mice $[17,18]$. Yet, these studies do not consider long-term storage and, more importantly for our studies, do not employ an electronic nose in their work. As the electronic nose does not detect individual chemicals and the sensitivity/selectivity of the sensors is very different-detecting both small molecular weight gases and large organics at the same time. Therefore, we set out to understand the rate of urinary degradation at $-80^{\circ} \mathrm{C}$ using the electronic nose and from this infer when degradation of the sample becomes significant. To undertake this study urine samples from patients with Type 2 Diabetes Mellitus (T2DM) were collected over a four and half year period and stored at $-80^{\circ} \mathrm{C}$. Though diabetic samples were used in this study (due to availability), it is likely that the overall degradation of urines from this group would be similar to other diseases. These samples were then analyzed using by FAIMS (field-asymmetric ion mobility spectrometry), as a type of electronic nose.

\section{Materials and Methods}

\subsection{Patients}

In diabetes mellitus, the deficiency of insulin alters the metabolism of carbohydrates, proteins and lipids, resulting in high glucose levels present in urine. Patients with type 2 diabetes (T2DM) were recruited from the metabolic clinic at University Hospital Coventry and Warwickshire (UHCW) NHS Trust between December 2009 and May 2014. In total, 87 samples were obtained over 53 months and tested as single batch of samples in May 2014 over the course of two weeks. The only criterion for inclusion was T2DM as defined by WHO criteria. Exclusion criteria included sepsis, concomitant diagnosis of inflammatory bowel disease, irritable bowel syndrome, coeliac disease or malignancy. 
Written informed consent was obtained from all individual participants patients included in the study. Patients were recruited as part of the FAMISHED study. Scientific and ethical approval was acquired from the local Research and Development Office as well as Warwickshire Ethical committee ref: 09/H1211/38. Demographic details are shown in Table 1.

Table 1. There were a total of 87 patients; with incomplete data on 15 patients hence this table demonstrates demographics on the remaining 72 patients. OHA—oral hypoglycaemic agents; $\mathrm{HbA1c}$ - glycated haemoglobin. HbA1C in normal subjects is $20-42 \mathrm{mmol} / \mathrm{mol}$.

\begin{tabular}{cccc}
\hline \multicolumn{2}{c}{ Demographic Data } & Diabetes Medication & Frequency \\
\hline Male (\%) & $44(61)$ & OHA & 39 \\
Female (\%) & $28(39)$ & Insulin & 7 \\
Mean age & 56 & OHA + Insulin & 18 \\
Median age & 59 & Nil & 8 \\
Mean BMI & 39 & HbA1c & $(\mathrm{mmol} / \mathrm{mol})$ \\
Median BMI & 39 & Mean HbA1c & 67 \\
& & Median Hb A1c & 57 \\
\hline
\end{tabular}

\subsection{Urine Collection, Storage and Transfer}

Urine samples from patients with T2DM at UHCW NHS Trust were collected at clinic and stored at $-80{ }^{\circ} \mathrm{C}$ within two hours of collection. They were transferred to the University of Warwick in a box of dry ice. The samples were then defrosted in a laboratory fridge at $3^{\circ} \mathrm{C}$ overnight prior to analysis.

\subsection{FAIMS Analysis}

For this study, a commercial FAIMS instrument was employed, specifically a Lonestar (Owlstone, UK). FAIMS (or sometimes called Differentially Mobility Spectrometry-DMS), has been seen as a useful means of separating gases and vapours since its conception in the 1980s [19]. They come in two basic configurations using either a cylindrical or (as in our case) or planar design [20]. Such technology has found favour in the security area for the detection of chemical warfare agents [21], but has been used for a range of other applications either on its own or in combination with mass-spectrometry [22-24]. FAIMS carries similar advantages in use to many electronic noses, it undertakes headspace analysis, it uses air as the carrier gas, is relatively portable, is simple to operate, costs a similar amount to existing commercial electronic noses and importantly, as it relies on a physical measurement of a chemical, it suffers less from drift/poisoning than most electronic nose instruments. Sensitivity strongly depends on proton affinity of the chemical of interest, but ranges between parts per million and parts per trillion [25] and in use compares well with techniques such as GCMS [26]. It is worth noting that FAIMS is far more sensitive than a standard GCMS system and the latter would require the use of a sophisticated pre-concentration system (such as absorbent tubes) to reach similar sensitivity levels. FAIMS is composed of three stages, ionization, filtration/separation and detection. In use, the sample headspace is drawn into the instrument (in our case using a dynamic sampling methodology) where it is ionized (Ni-63 source) and mixed with a carrier gas of clean/dry air. The total sample is then pushed between two separator plates to which an oscillating asynchronous waveform is applied (up to $\mathrm{GHz}$, with an amplitude up to hundreds of volts). The plates are subjected to a high positive potential for a short period of time, followed by a small in magnitude negative potential for a much longer period of time, but where the amplitude $x$ time is the same in both cases. The Lonestar uses a silicon based separator with a sub $50 \mu \mathrm{m}$ electrode spacing to create very high electric fields of up to $100 \mathrm{kV} / \mathrm{cm}$. In use, the short/high potential is ramped through a series of values, described as the "dispersion field" from 0 up to the systems maximum value. Ionised molecules that enter the separation stage are then attracted, repelled or not affected by the electric field, depending upon the mobility difference between high- and low-field regime (as shown in Figure 1). Any ion that touches one of the separator plates, loses its charge and is not detected. However, those ions that pass between the plates then exit the system where they collide with a detection plate and their charge measured. To increase the 
chemical detection range, a DC compensation voltage is applied to the plates, which is used to remove the ion movement towards one of the plates. This compensation voltage (normally a single figure voltage) is swept between a positive and negative potential and therefore for a certain compensation voltage, only chemicals with a specific differential mobility will be detected. The mobility of an ion depends on the mass, charge, size and shape since the field and the velocity are constant and are related by the equation below.

$$
v=K E
$$

where $v$ and $K$ are the velocity and mobility of ion respectively and $E$ is the electric field strength. Since velocity changes is not proportional to the electric field intensity variations, at higher electric fields the ion mobility can be expressed by the following equation.

$$
K_{h}=K\left[1+\alpha\left(\frac{E}{N}\right)^{2}+\beta\left(\frac{E}{N}\right)^{4}\right]
$$

where $K_{h}$ and $K$ are the high-field mobility and low-field mobility respectively. $\alpha$ and $\beta$ are compound specific values which account for high-field mobility effect, $N$ is carrier gas density number and $E$ is the electric field strength. More details of this process can be found in $[25,27]$.

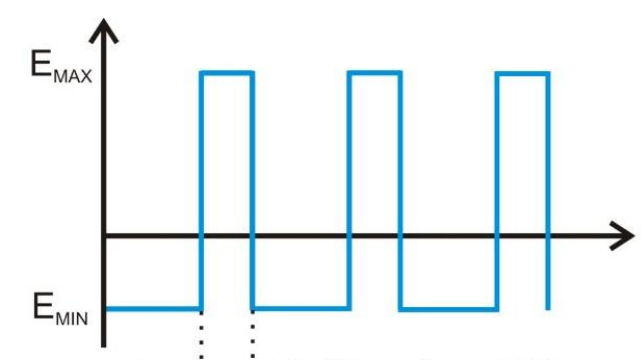

Asymmetric Waveform $\mathrm{V}(\mathrm{t})$

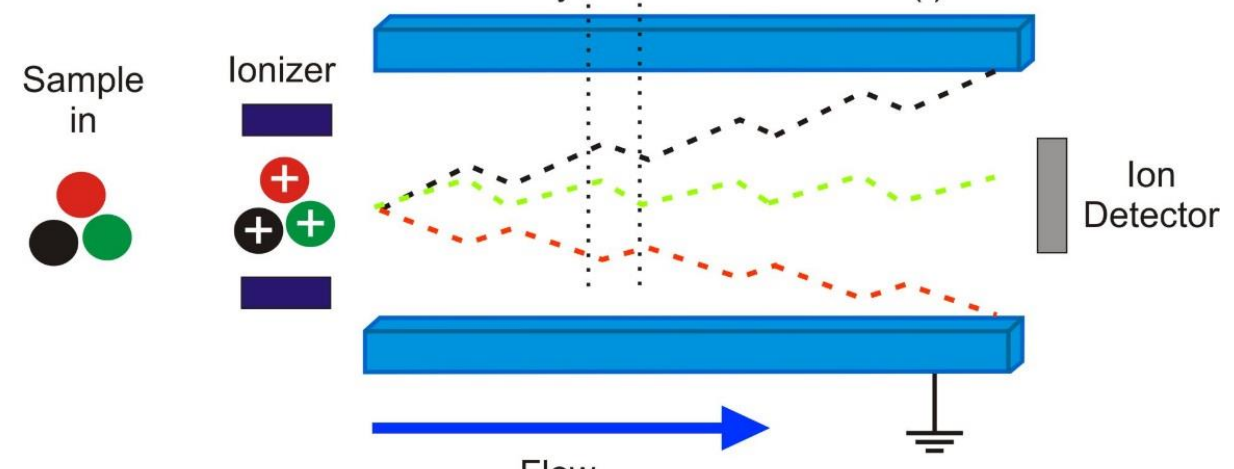

Flow

Figure 1. Asymmetric waveform applied in field-asymmetric ion mobility spectrometry (FAIMS) and ions being separated by an asymmetric waveform. $\mathrm{E}_{\mathrm{MAX}}$ and $\mathrm{E}_{\mathrm{MIN}}$ are the differences in generated electric fields to the applied waveform $\mathrm{V}(\mathrm{t})$. The coloured lines between the plates show an example of possible paths depending on the molecules differential mobility.

\subsection{Analysis Methodology}

$5 \mathrm{~mL}$ of urine were aliquoted from each sample into a $10 \mathrm{~mL}$ glass vial and placed into an ATLAS sample system (Owlstone, UK) attached to the front of the FAIMS, (Lonestar, Owlstone, UK). The ATLAS is a dynamic headspace sampler system that controls the sample temperature, sample agitation and the flow rate over the sample. This setup is commonly used for samples of this type by our group $[10,11]$. This heated the sample to $40 \pm 0.1^{\circ} \mathrm{C}$. Each sample was tested three times sequentially, with each run having a flow rate over the sample of $200 \mathrm{~mL} / \mathrm{min}$ of clean dry air. Further make-up air was added to create a total flow rate of $2 \mathrm{~L} / \mathrm{min}$. The FAIMS was scanned from 0 to $99 \%$ dispersion field in 51 steps, $-6 \mathrm{~V}$ to $+6 \mathrm{~V}$ compensation voltage in 512 steps and both positive and negative ions were detected to create a test file composed of 52,224 data points. Figure 2 shows 
our experimental setup, with Figure 3 showing a typical output for a diabetic urine sample (positive ions only).

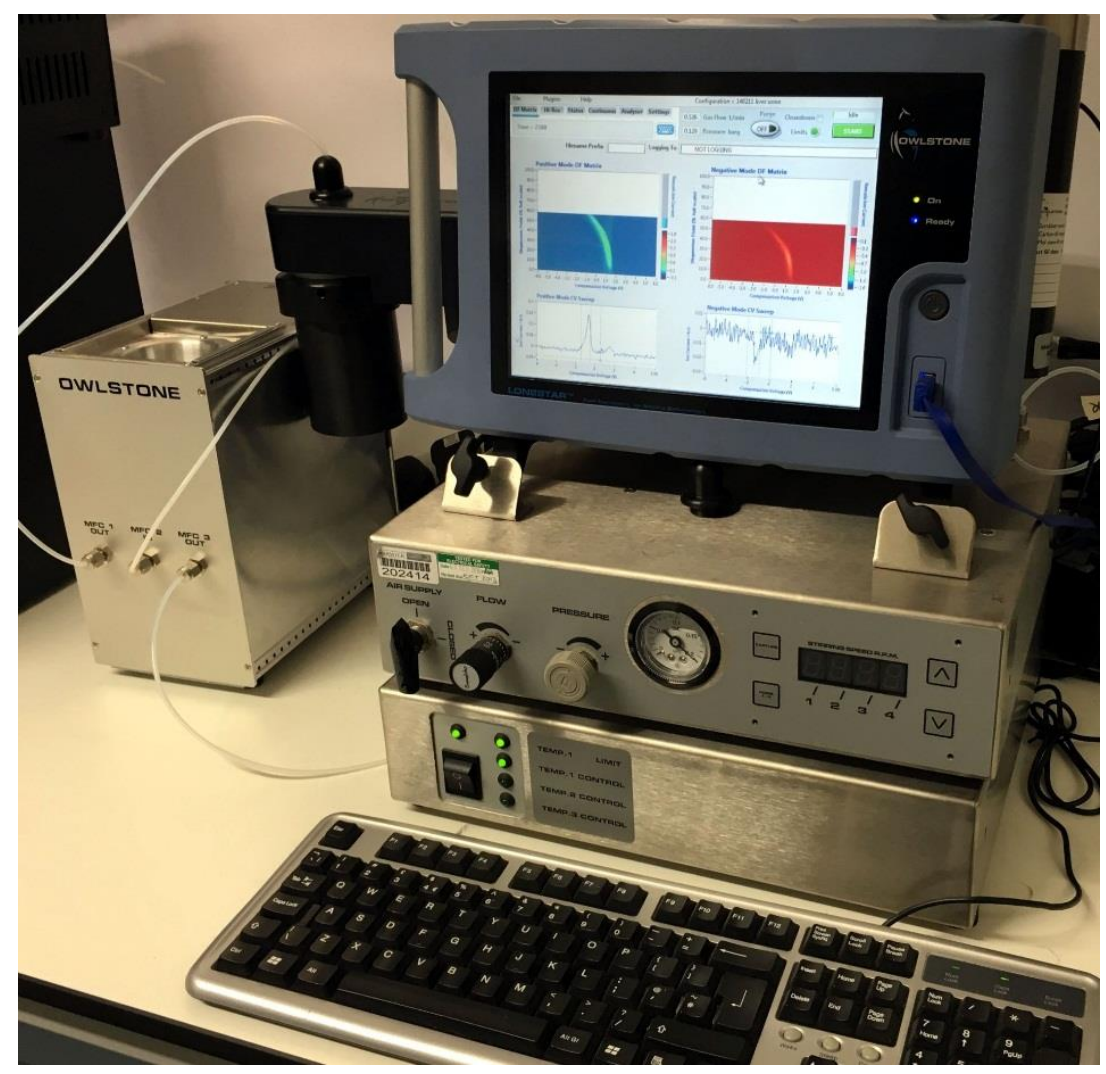

Figure 2. FAIMS experimental setup with Lonestar and ATLAS sampling system.

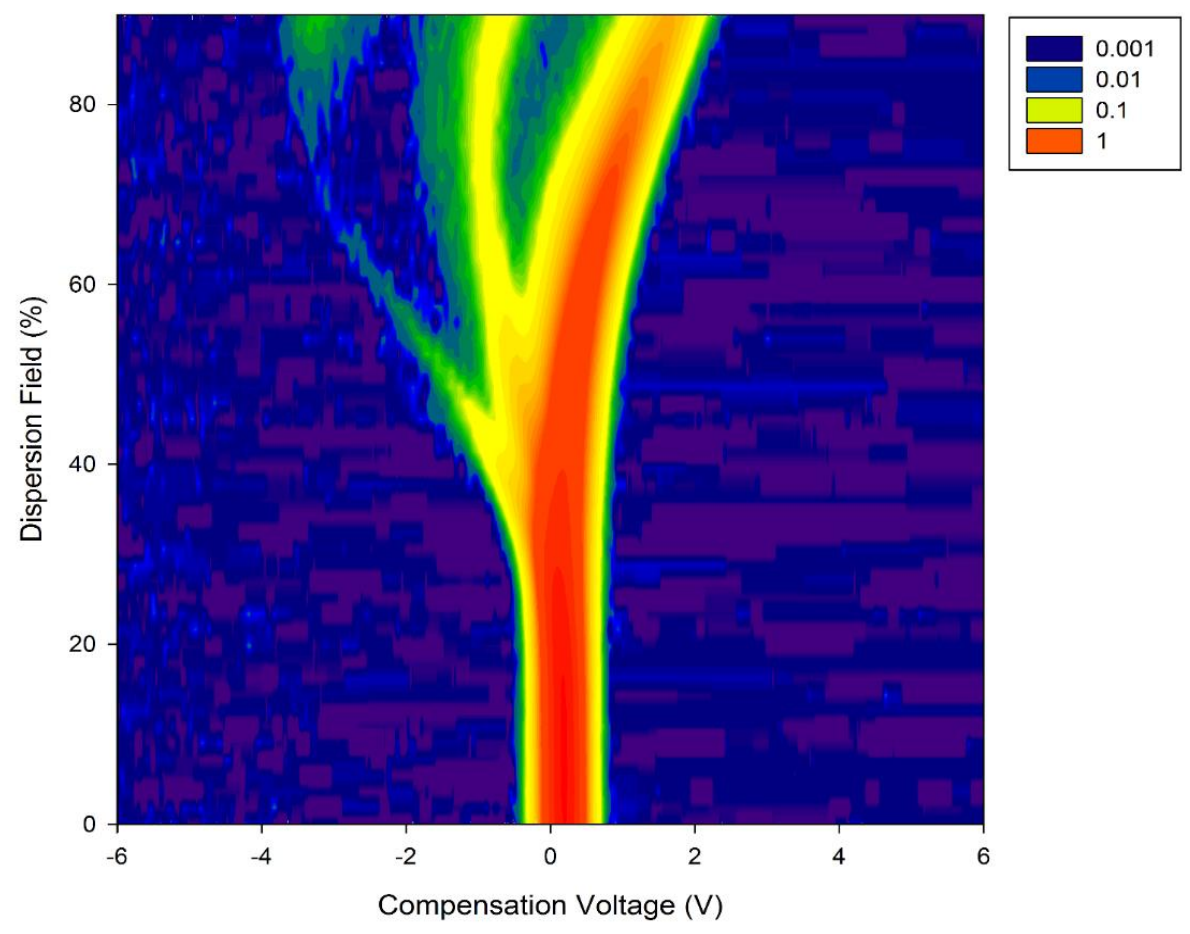

Figure 3. Typical FAIMS output to a diabetic urine sample. 


\section{Results and Discussion}

\subsection{Results}

In our initial analysis we considered the total chemical concentration (a mixture of gases and volatile organic compounds). In FAIMS this is easily achieved by summing up all of the values at a zero dispersion field (hence no separation). Figure 4 demonstrates the changes in urinary gas/volatile concentration between 2009 and 2014, using this data. The graph shows that the total amount of chemicals released decreases with sample age with more modern samples having a higher output. This trend is confirmed in both the positive and negative electric fields of the FAIMS instrument depicted by the red and green dots, respectively. We have also averaged the total output of positive and negative ions to emphasis the changes over time, as shown in Table 2. It is worth noting that as the samples get much older (greater than 3 years), the output increases once more, with an associated increase in variance between the samples.

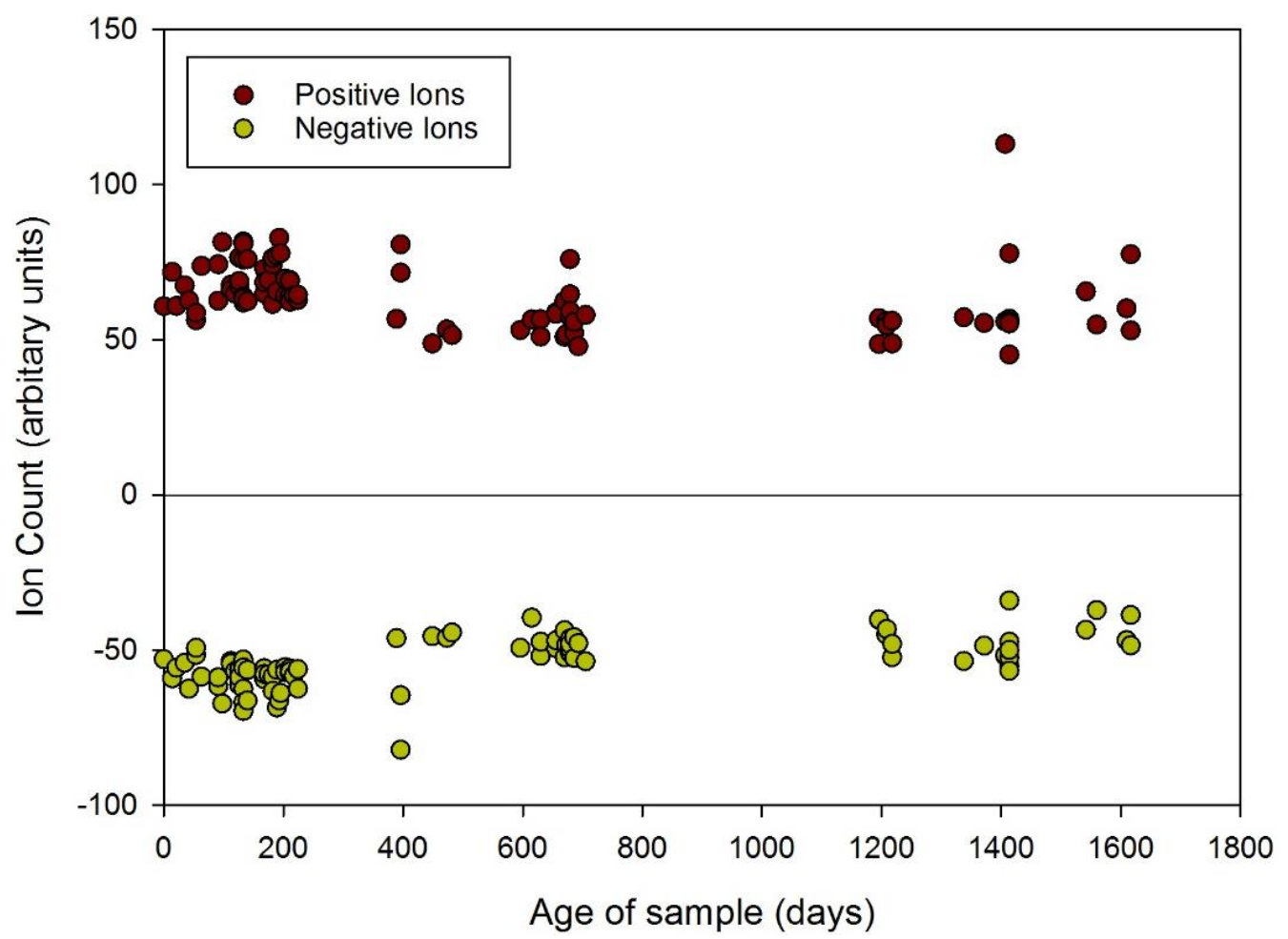

Figure 4. Change in total number of urinary volatile organic compounds over time (from December 2009 to May 2014).

In addition to measuring the change in overall gas/volatile concentration, we also investigated the chemical diversity of the samples. We define chemical diversity as a measure of the amount of different chemicals in the sample, independent of concentration. As FAIMS separates chemicals based on mobility, it is possible to measure the diversity of chemicals at a specific dispersion field, though we are unable to separate chemicals with the same differential mobility. In our case, we took a 50\% dispersion field (selected as a compromise between chemical separation and measuring as many chemical as possible), then used a threshold value of 0.01 to remove the background noise. This result is shown in Figure 5. Again this result has been averaged into each year and is shown in Table 2. 
Table 2. Changes in output signal for both positive and negative ions as a function of time. Percentage for year one taken as $100 \%$.

\begin{tabular}{ccccccc}
\hline \multicolumn{7}{c}{ Positive Ions (Arbitrary Units) } \\
\hline Year & 2014 & 2013 & 2012 & 2011 & 2010 & 2009 \\
Age (days) & $0-147$ & $148-503$ & $504-859$ & $860-1215$ & $1216-1572$ & $1572-1617$ \\
Average & 68.2 & 67.0 & 57.0 & 53.4 & 62.3 & 63.4 \\
s.d. & 7.4 & 8.6 & 6.5 & 3.8 & 17.7 & 12.6 \\
\% & 100.0 & 98.2 & 83.6 & 78.3 & 91.3 & 93.0 \\
\hline \multicolumn{7}{c}{ Negative Ions (Arbitrary Units) } \\
\hline Year & 2014 & 2013 & 2012 & 2011 & 2010 & 2009 \\
Age (days) & $0-147$ & $148-503$ & $504-859$ & $860-1215$ & $1216-1572$ & $1572-1617$ \\
Average & -58.4 & -58.2 & -48.5 & -44.9 & -48.6 & -44.7 \\
s.d. & 5.3 & 7.8 & 3.5 & 4.7 & 7.0 & 5.2 \\
\% & 100.0 & 99.7 & 83.0 & 76.9 & 83.2 & 76.5 \\
\hline \multicolumn{7}{c}{ Threshold Values (Positive Ions Only) } \\
\hline Year & 2014 & 2013 & 2012 & 2011 & 2010 \\
Age (days) & $0-147$ & $148-503$ & $504-859$ & $860-1215$ & $1216-1572$ & $1572-1617$ \\
Average & 54.3 & 55.5 & 53.2 & 54.3 & 52.8 & 52.3 \\
s.d. & 2.3 & 2.2 & 2.2 & 1.8 & 2.1 & 1.5 \\
\% & 100.0 & 102.2 & 98.0 & 100 & 97.2 & 96.3 \\
\hline
\end{tabular}

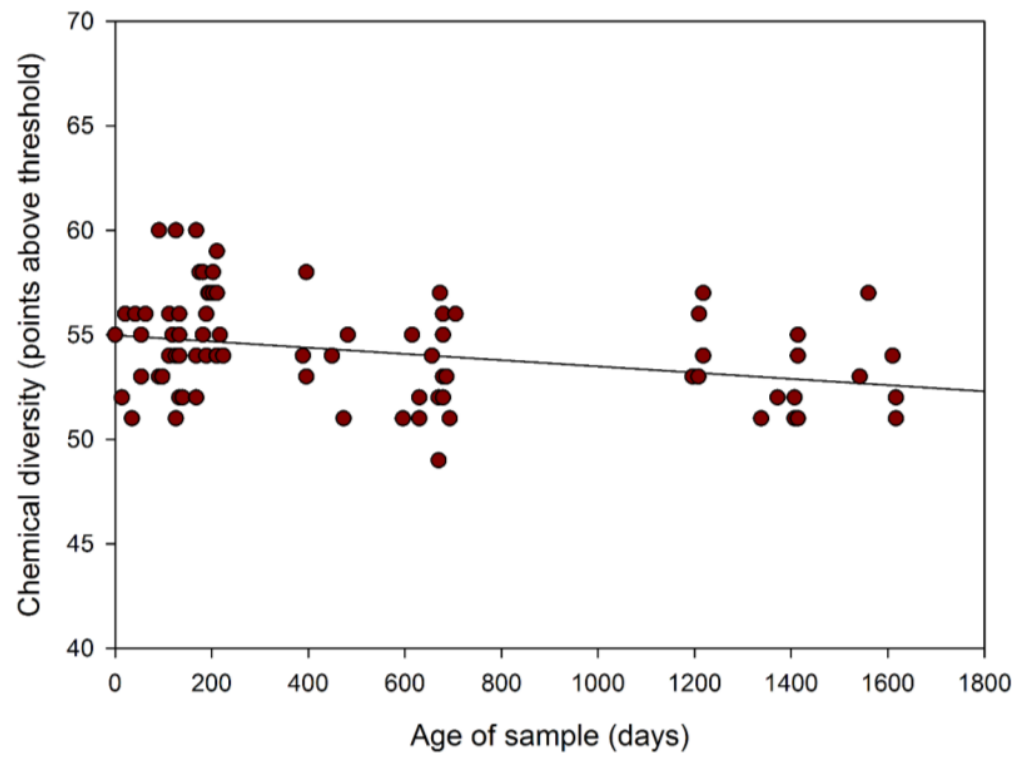

Figure 5. Chemical diversity of urinary volatile organic compounds over time (from December 2009 to May 2014), with linear fit to emphasis output change.

Of note, there is less variation in the total number of emissions in the more recent samples with greater uniformity and stability of concentrations together with tighter clustering of the total number of chemicals released. This is particularly evident for the urine samples obtained from October 2013 to May 2014, which demonstrates that the chemical information is stable for at least 9 months, as shown in Figure 6 and Table 3. In the latter table, the data for 12 months is included and after a 9 month period there data suggests a fall-off in chemical output. Some care should be taken due to the variance in measurements, but there is a clear reduction in chemical output after 9 months. 


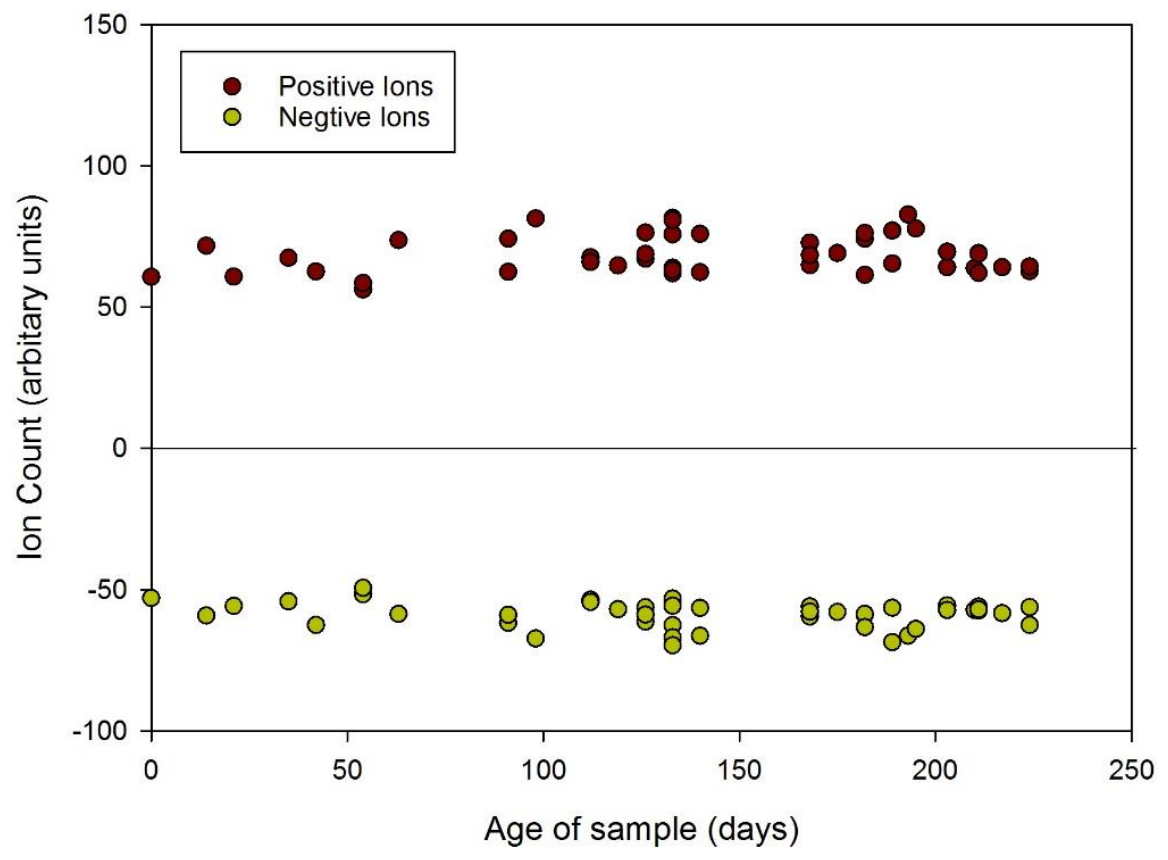

Figure 6. Change in total number of urinary VOCs over time (from May 2014 to September 2014).

Table 3. Total positive ion counts for samples collected in 2014 in 3 monthly periods.

\begin{tabular}{cccccc}
\hline Year & $\begin{array}{c}\text { May 2014- } \\
\text { March 2014 }\end{array}$ & $\begin{array}{c}\text { February 2014- } \\
\text { December 2013 }\end{array}$ & $\begin{array}{c}\text { November 2013- } \\
\text { Septeber 2013 }\end{array}$ & $\begin{array}{c}\text { August 2013- } \\
\text { June 2013 }\end{array}$ & $\begin{array}{c}\text { May 2013- } \\
\text { March 2013 }\end{array}$ \\
\hline Age (days) & $0-88$ & $89-178$ & $179-269$ & $270-356$ & $357-445$ \\
Average & 64.0 & 70.0 & 69.0 & No data & 64.4 \\
s.d. & 6.3 & 6.8 & 6.7 & No data & 12.1 \\
\hline
\end{tabular}

\subsection{Discussion}

In this paper we set out to understand how the gases and vapours emanating from a stored urine sample are affected by storage time, using the electronic nose. In these experiments diabetes samples were used, which were collected over an extended period of time. In most cases samples would be tested well before this four and a half year period, but it is common to store blood and other samples for many years for future researchers. Our study suggests that this is not feasible when dealing with urine samples for gas analysis. It is likely that the loss of chemical signal would be associated with a specific disease, however here we only considered one disease-diabetes and we did not track biomarkers as the sample ages. Furthermore, it is very unlikely that individual groups would undertake this type of study for each disease, making this an interesting result and could guide other researchers. It is also likely that other biological samples (such as blood and stool) would also degrade at $-80^{\circ} \mathrm{C}$ and further work is needed to understand this process.

The loss of gases and volatiles from a stored urine sample is not totally unforeseen. The results indicate that we are losing chemical information—both the amount of signal available and the diversity. The latter is not unexpected as when chemical concentration reduces, some chemicals will then fall below the detection limit of the instrument. As interesting, when the samples get very old, their emissions become very erratic, suggestion some secondary breakdown has occurred in some samples, significantly increasing the total emissions.

The reason for this is loss of signal is not totally clear, however it is well known that all gases and vapours will emanate from a sample over time as equilibrium is reached between the urine and the airspace in the storage container. This process can be reduced at $-80^{\circ} \mathrm{C}$, but is not halted, especially 
for low molecular weight gases dissolved in the sample. In addition, we may be observing water in the urine evaporating as it ages with the subsequent release of water-soluble volatiles. For the very old samples, we may be seeing bacterial activity that could be resulting in a higher chemical output, as described in [8]. Finally, in our case, the samples are stored in standard plastic sterilin bottles, commonly used by the medical profession. It may be possible that the plastic is absorbing volatiles over time. This requires further investigation, but beyond the scope of this study.

From our experiments, we can tentatively propose a urine sample best before date of 12 months, though ideally samples should be tested within 9. Analysis of the results shows that there is no significant loss in chemicals over this 9 month period, but appears to increase relatively rapidly after this. However, care should still be taken to ensure that when samples are collected from different groups (disease and control) then samples should be collected at similar times and not be separated by a significant period. We have found (unpublished results) that even fresh samples vs. 6 month old samples could result in separation (using pattern recognition techniques) based on age instead of disease.

Of final note, for samples taken over the first 9 months, the variation in chemical output and the differences in chemical diversity only changes by around $10 \%$ across the samples. The diabetic patients were under no dietary controls and urines were collected as spot samples. Though the samples were from a single disease group, it is interesting that the differences in gender, age and diet only produced a relatively small change in volatile output.

\section{Conclusions}

Gas phase biomarkers are becoming of ever increasing interest to researchers. One instrument showing promise is the electronic nose-an instrument that attempts to mimic the biological olfactory system by analyzing samples as a whole. Urine is an important sample type, due to its ease of collection and clinical utility. However, when undertaking this type of research, the question of long-term stability in storage becomes important, especially for diseases with low prevalence. Although in this study samples were taken and analysed from those with diabetes and we did not track any specific biomarker (which is difficult with the electronic nose), it is biologically plausible that these results could be used to infer for diabetes and other medical conditions a possible storage time. Our results suggest that even at $-80^{\circ} \mathrm{C}$, changes of total chemical concentrations are noticeable before 200 days, and continue over time. Extrapolating our results would suggest that storage at $-20{ }^{\circ} \mathrm{C}$ would result in more rapid loss of chemicals but remains uncertain at which time point the stored sample loses its diagnostic value.

Acknowledgments: The authors would like to thank all the patients from UHCW NHS Trust who participated in the study. The authors would also like to acknowledge the BRET (Bardhan Research Education Trust) charity, which has supported funding for materials and equipment costs. The funders had no role in study design, data collection and analysis, decision to publish, or preparation of the manuscript.

Author Contributions: James A. Covington, Karna D. Bardhan and Ramesh P. Arasaradnam conceived and designed the experiments; James A. Covington and Siavash Esfahani performed the experiments; James A. Covington, Siavash Esfahani and Nidhi M. Sagar analysed the data; Ioannis Kyrou, Nicola O'Connell, Karna D. Bardhan, Chuka Nwokolo, Ella Mozdiak, James A. Covington contributed materials/analysis tools; James A. Covington, Siavash Esfahani and Nidhi M. Sagar wrote the manuscript.

Conflicts of Interest: The authors declare no conflict of interest.

\section{References}

1. Persaud, K.; Dodd, G. Analysis of discrimination mechanisms in the mammalian olfactory system using a model nose. Nature 1982, 299, 352-355. [CrossRef] [PubMed]

2. Kateb, B.; Ryan, M.A.; Homer, M.L.; Lara, L.M.; Yin, Y.; Higa, K.; Chen, M.Y. Sniffing out cancer using the JPL electronic nose: A pilot study of a novel approach to detection and differentiation of brain cancer. NeuroImage 2009, 47, T5-T9. [CrossRef] [PubMed]

3. Bruins, M.; Rahim, Z.; Bos, A.; van de Sande, W.W.; Endtz, H.P.; van Belkum, A. Diagnosis of active tuberculosis by e-nose analysis of exhaled air. Tuberculosis 2013, 93, 232-238. [CrossRef] [PubMed] 
4. Byun, H.G.; Persaud, K.C.; Pisanelli, A.M. Wound-state monitoring for burn patients using E-Nose/SPME system. ETRI J. 2010, 32, 440-446. [CrossRef]

5. Arasaradnam, R.P.; Covington, J.A.; Harmston, C.; Nwokolo, C.U. Review article: Next generation diagnostic modalities in gastroenterology-gas phase volatile compound biomarker detection. Aliment. Pharmacol. Ther. 2014, 39, 780-789. [CrossRef] [PubMed]

6. Wilson, A.D.; Baietto, M. Advances in electronic-nose technologies developed for biomedical applications. Sensors 2011, 11, 1105-1176. [CrossRef] [PubMed]

7. Covington, J.A.; der Schee, M.V.; Edge, A.S.L.; Boyle, B.; Savage, R.S.; Arasaradnam, R.P. The application of FAIMS gas analysis in medical diagnostics. Analyst 2015, 140, 6775-6781. [CrossRef] [PubMed]

8. Montuschi, P.; Mores, N.; Trové, A.; Mondino, C.; Barnes, P.J. The electronic nose in respiratory medicine. Respiration 2013, 85, 72-84. [CrossRef] [PubMed]

9. D'Amico, A.; di Natale, C.; Falconi, C.; Martinelli, E.; Paolesse, R.; Pennazza, G.; Santonico, M.; Sterk, P.J. Detection and identification of cancers by the electronic nose. Expert Opin. Med. Diagn. 2013, 6, 175-185. [CrossRef] [PubMed]

10. Covington, J.A.; Westenbrink, E.W.; Ouaret, N.; Harbord, R.; Bailey, C.; O'Connell, N.; Cullis, J.; Williams, N.; Nwokolo, C.U.; Bardhan, K.D.; et al. Application of a novel tool for diagnosing bile acid diarrhoea. Sensors 2013, 13, 11899-11912. [CrossRef] [PubMed]

11. Arasaradnam, R.P.; Ouaret, N.; Thomas, M.G.; Quraishi, N.; Heatherington, E.; Nwokolo, C.U.; Bardhan, K.D.; Covington, J.A. A novel tool for noninvasive diagnosis and tracking of patients with inflammatory bowel disease. Inflamm. Bowel Dis. 2013, 19, 999-1003. [CrossRef] [PubMed]

12. Sykes, B.D. Urine stability for metabolomic studies: effects of preparation and storage. Metabolomics 2007, 3, 19-27.

13. Gika, H.G.; Theodoridis, G.A.; Wilson, I.D. Liquid chromatography and ultra-performance liquid chromatography-mass spectrometry fingerprinting of human urine: Sample stability under different handling and storage conditions for metabonomics studies. J. Chromatogr. A 2008, 1189, 314-322. [CrossRef] [PubMed]

14. Dunn, W.B.; Broadhurst, D.; Ellis, D.I.; Brown, M.; Halsall, A.; O’Hagan, S.; Spasic, I.; Tseng, A.; Kell, D.B. A GC-TOF-MS study of the stability of serum and urine metabolomes during the UK Biobank sample collection and preparation protocols. Int. J. Epidemiol. 2008, 37, i23-i30. [CrossRef] [PubMed]

15. Smith, S.; Burden, H.; Persad, R.; Whittington, K.; de Lacy Costello, B.; Ratcliffe, N.M.; Probert, C.S. A comparative study of the analysis of human urine headspace using gas chromatography-mass spectrometry. J. Breath Res. 2008, 2. [CrossRef] [PubMed]

16. Huang, J.; Kumar, S.; Abbassi-Ghadi, N.; Španěl, P.; Smith, D.; Hanna, G.B. Selected ion flow tube mass spectrometry analysis of volatile metabolites in urine headspace for the profiling of gastro-esophageal cancer. Anal. Chem. 2013, 85, 3409-3416. [CrossRef] [PubMed]

17. Kwak, J.; Grigsby, C.C.; Smith, B.R.; Rizki, M.M.; Preti, G. Changes in volatile compounds of human urine as it ages: Their interaction with water. J. Chromatogr. B 2013, 941, 50-53. [CrossRef] [PubMed]

18. Kwak, J.; Grigsby, C.C.; Preti, G.; Rizki, M.M.; Yamazaki, K.; Beauchamp, G.K. Changes in volatile compounds of mouse urine as it ages: Their interactions with water and urinary proteins. Physiol. Behav. 2013, 120, 211-219. [CrossRef] [PubMed]

19. Buryakov, I.A.; Krylov, E.V.; Makas, A.L.; Nazarov, E.G.; Pervukhin, V.V.; Rasulev, U.K. Ion division by their mobility in high-tension alternating electric field. Sov. Tech. Phys. Lett. 1991, 17, 446-447.

20. Guevremont, R. High-field asymmetric waveform ion mobility spectrometry: A new tool for mass spectrometry. J. Chromatogr. A 2004, 1058, 3-19. [CrossRef]

21. Guo, D.; Wang, Y.; Li, L.; Wang, X.; Luo, J. Precise determination of nonlinear function of ion mobility for explosives and drugs at high electric fields for microchip FAIMS. J. Mass Spectrom. 2015, 50, 198-205. [CrossRef] [PubMed]

22. Wang, Q.; Xie, Y.F.; Zhao, W.J.; Li, P.; Qian, H.; Wang, X.Z. Rapid microchip-based FAIMS determination of trimethylamine, an indicator of pork deterioration. Anal. Methods 2014, 6, 2965-2972. [CrossRef]

23. Xia, Y.Q.; Steven, W.T.; Mohammed, J. LC-FAIMS-MS/MS for quantification of apeptide in plasma and evaluation of FAIMS global selectivity from plasma components. Anal. Chem. 2008, 80, 7137-7143. [CrossRef] [PubMed] 
24. Lambertus, G.R.; Fix, C.S.; Reidy, S.M.; Miller, R.A.; Wheeler, D.; Nazarov, E.; Sacks, R. Silicon microfabricated column with microfabricated differential mobility spectrometer for GC analysis of volatile organic compounds. Anal. Chem. 2005, 77, 7563-7757. [CrossRef] [PubMed]

25. Wilks, A.; Hart, M.; Koehl, A.; Somerville, J.; Boyle, B.; Ruiz-Alonso, D. Characterization of a miniature, ultra-high-field, ion mobility spectrometer. Int. J. Ion Mobil. Spectrom. 2012, 15, 199-222. [CrossRef]

26. Gabryelski, W.; Wu, F.; Froese, K.L. Comparison of high-field asymmetric waveform ion mobility spectrometry with GC methods in analysis of haloacetic acids in drinking water. Anal. Chem. 2003, 75, 2478-2486. [CrossRef] [PubMed]

27. Krylov, E.V. Comparison of the planar and coaxial field asymmetrical waveform ion mobility spectrometer (FAIMS). Int. J. Mass Spectrom. 2003, 225, 39-51. [CrossRef]

(C) 2016 by the authors; licensee MDPI, Basel, Switzerland. This article is an open access article distributed under the terms and conditions of the Creative Commons by Attribution (CC-BY) license (http://creativecommons.org/licenses/by/4.0/). 